\title{
Streptococcus gallolyticus subsp. pasteurianus: un agente inusual ocasionando una infección común
}

\author{
Marcela Víquez-Víquez¹, María G. Brenes-Meléndez², Constanza Chacón-González ${ }^{1 *} y$ \\ María L. Ávila-Agüero ${ }^{2,3}$ \\ ${ }^{1}$ Universidad de Ciencias Médicas; ${ }^{2}$ Hospital Nacional de Niños, Caja Costarricense de Seguro Social; ${ }^{3}$ Universidad de Costa Rica. San José, \\ Costa Rica
}

\begin{abstract}
Resumen
Introducción: Streptococcus gallolyticus subsp. pasteurianus es un coco grampositivo, catalasa negativo, perteneciente al grupo $D$ de Lancefield, que se encuentra relacionado con cuadros de sepsis neonatal, especificamente con meningitis. Caso clínico: Se reporta el caso de un paciente de 2 meses con un cuadro de meningitis por S. gallolyticus subsp. pasteurianus. Se describen la sintomatología y el abordaje diagnóstico y terapéutico. Asimismo, se realiza una revisión de la literatura que evidencia el subdiagnóstico de este agente como causa de meningitis en pediatría. Conclusiones: Este caso representa el primer aislamiento de este agente en el Hospital Nacional de Niños de Costa Rica, de la Caja Costarricense de Seguro Social.
\end{abstract}

Palabras clave: Meningitis. Pediatría. Sepsis. Streptococcus gallolyticus.

\section{Streptococcus gallolyticus subsp. pasteurianus: a common infection caused by an unusual agent}

\begin{abstract}
Background: Streptococcus gallolyticus subsp. pasteurianus is a positive Gram stain, catalase negative coccus that belongs to the Lancefield group D. It is associated with clinical manifestations such as neonatal sepsis and meningitis. Case report: The case of a 2-month-old male patient with meningitis caused by S. gallolyticus subsp. pasteurianus is described. Moreover, the symptoms, diagnosis, and management of the patient are discussed. Also, a review of the literature was conducted showing the sub-diagnosis of this agent as a cause of meningitis in pediatrics. Conclusions: This case represents the first detection of this agent in the National Children's Hospital of Costa Rica, which is part of the Caja Costarricense de Seguro Social.
\end{abstract}

Key words: Meningitis. Pediatrics. Sepsis. Streptococcus gallolyticus.

Correspondencia:

*Constanza Chacón-González

E-mail: constanzachacong@gmail.com

(http://creativecommons.org/licenses/by-nc-nd/4.0/).
Bol Med Hosp Infant Mex. 2021;78(2):148-151

www.bmhim.com 


\section{Introducción}

Streptococcus gallolyticus, conocido previamente como Streptococcus bovis, es un coco grampositivo, catalasa negativo, que reacciona con los anticuerpos para el grupo D de Lancefield. Se diferencia de los Enterococcus por su intolerancia al cloruro de sodio y la ausencia de la enzima pirrolidonil arilamidasa. Existen tres subespecies que causan patología en el ser humano: gallolyticus, infantarius y pasteurianus ${ }^{1}$.

Anteriormente, S. bovis se subdividía en biotipo I y II, según su capacidad de fermentar el manitol. Lo que hoy se conoce como S. gallolyticus subsp. gallolyticus era S. bovis biotipo I, mientras que S. gallolyticus subsp. infantarius y pasteurianus eran S. bovis biotipos II.1 y II.2, respectivamente. La importancia clínica de estos cambios taxonómicos en pediatría yace en que las infecciones por subespecies de $S$. gallolyticus se manejan de forma distinta a las infecciones por Enterococcus ${ }^{1-5}$.

S. gallolyticus subsp. gallolyticus se ha asociado principalmente con bacteriemia y endocarditis, y con menor frecuencia con meningitis e infecciones óseas $y$ articulares en adultos ${ }^{6}$. Este patógeno no se ha estudiado de manera tan extensa en pediatría, y los estudios en los que se ha identificado a nivel de subespecie en niños son raros. La literatura solo reporta casos esporádicos ${ }^{7,8}$.

S. gallolyticus subsp. pasteurianus fue reconocido desde el año 2000 como causa de meningitis neonatal. En el mundo se han reportado casos de meningitis y de sepsis neonatal por este agente ${ }^{9,10}$. En América Latina son pocos los datos referentes a esta presentación clínica en pediatría ${ }^{11}$ y los casos de endocarditis neonatal son escasos ${ }^{12}$.

El objetivo de este artículo es reportar el primer aislamiento positivo de $S$. gallolyticus como causa de meningitis en un niño de 2 meses en el Hospital Nacional de Niños, de la Caja Costarricense de Seguro Social, en Costa Rica, y así contribuir a la literatura latinoamericana sobre casos similares, para dar a conocer estos aislamientos esporádicos, poder sospecharlos y evitar su subdiagnóstico.

\section{Caso clínico}

Se describe el caso de un paciente de sexo masculino de 2 meses y 8 días. Se trata de un recién nacido de término adecuado para la edad gestacional, sin antecedentes perinatales ni patológicos de importancia, y vacunas al día según el esquema nacional de vacunación. Acudió a la consulta por un cuadro súbito
- de horas de evolución - caracterizado por fiebre, rechazo del alimento, irritabilidad, somnolencia y vómitos en proyectil. Sin historia de apneas, crisis de cianosis ni convulsiones. No mostró nexo epidemiológico positivo.

En la exploración física se encontró irritable, hipotenso, taquicárdico, taquipneico, con piel marmórea y datos de hipoperfusión, por lo que ameritó reanimación con líquidos. Se documentó una fontanela anterior abombada y un puntaje de la Escala de Glasgow modificada de 12 puntos. No se documentaron signos meníngeos. Se solicitaron estudios microbiológicos de líquido cefalorraquídeo (LCR), sangre y orina, y se inició cobertura antibiótica empírica con cefotaxima y ampicilina.

Los resultados del análisis del LCR fueron los siguientes: albúmina, $298 \mathrm{mg} / \mathrm{dl}$; glucosa, indetectable; microproteínas, $431 \mathrm{mg} / \mathrm{dl}$; eritrocitos, 180; leucocitos, 9,860; polimorfonucleares, $90 \%$, y mononucleares, $10 \%$. El análisis microbiológico de la muestra de LCR evidenció la presencia de S. gallolyticus subsp pasteurianus, el cual se identificó por medio de métodos automatizados (Vitek 2 Compact $\circledast$, bioMérieux). Después de 33 horas, dos hemocultivos periféricos de sitios diferentes fueron positivos para Enterococcus faecalis, aunque S. gallolyticus no se detectó en ellos. No se aislaron patógenos en el urocultivo.

A pesar de la cobertura antibiótica inicial, el paciente inició con picos febriles, deterioro clínico, palidez, piel marmórea e irritabilidad al tercer día de tratamiento. La exploración física no mostró signos meníngeos y el paciente conservaba los reflejos primitivos para la edad. Se solicitó una valoración del paciente por infectología, quienes decidieron modificar la cobertura empírica e iniciar penicilina sódica y gentamicina.

Ninguno del resto de los exámenes complementarios realizados al paciente demostró hallazgos patológicos (ultrasonido cerebral, emisiones otoacústicas, ecocardiograma y ultrasonido de abdomen y de vías urinarias).

El paciente cursó estable, con adecuada evolución clínica y cultivos negativos a los 9 días desde su ingreso. Egresó luego de 14 días de tratamiento, sin complicaciones asociadas y con citas de control ambulatorio.

\section{Discusión}

El grupo $D$ de los estreptococos ha sufrido múltiples modificaciones taxonómicas en los últimos años. Originalmente se clasificaban en S. bovis, S. equinus, $S$. faecalis y $S$. faecium, que compartían la característica de presentar actividad contra antígenos de hidratos de carbono. 
La mayoría de los casos de infección por S. gallolyticus se han descrito en adultos, mientras que la literatura es limitada y poco clara en la población pediátrica. La bacteriemia y la endocarditis infecciosa son las dos presentaciones clínicas más frecuentes de $S$. gallolyticus subsp. gallolyticus en los adultos. Solo se ha reportado un caso de endocarditis neonatal por S. gallolyticus subsp. pasteurianus ${ }^{12}$. También se han reportado asociaciones entre pacientes con alteraciones del parénquima hepático y de la vesícula biliar y aislamientos de $S$. gallolyticus subsp. infantarius. Estos patógenos se han aislado en el 2-12\% de las heces de individuos sanos y en mayor porcentaje en personas con tumores colorrectales ${ }^{10}$, aunque también existen otras formas infecciosas menos comunes (articulares y óseas, peritonitis y del tracto urinario) ${ }^{1}$.

Se desconoce con exactitud la fisiopatología de S. gallolyticus subsp. pasteurianus como causante de meningitis y sepsis neonatal. Varias teorías han tratado de explicar cómo ocurre la infección, pero ninguna ha logrado sustentarse con evidencia. Se ha descrito una posible ruta ascendente como puerta de entrada, así como el contagio periparto por vía vaginal o tras el nacimiento a través de fómites o de la leche materna ${ }^{13}$.

La presentación clínica de la meningitis por S. gallolyticus subsp. pasteurianus suele ocurrir después de la primera semana de vida, mientras que es más común que la bacteriemia sea de inicio temprano. El cuadro es similar al producido por los estreptococos del grupo B (Streptococcus agalactiae), y como clínicamente son indistinguibles, se cree que existe un subdiagnóstico de casos ${ }^{1}$. La bacteriemia se asocia con dificultad respiratoria aguda y un cuadro compatible con sepsis neonatal, incluyendo síntomas neurológicos, irritabilidad y rechazo del alimento ${ }^{3}$.

En relación con lo mencionado, el paciente descrito inició de forma súbita con síntomas meníngeos, por lo que se ingresó para el manejo de un cuadro sugestivo de sepsis por meningitis bacteriana. Se decidió iniciar la cobertura antibiótica empírica con cefotaxima y ampicilina, dado que el protocolo para niños menores de 3 meses con datos de sepsis busca cubrir gérmenes - provenientes tanto del periodo neonatal como de la comunidad- que puedan coexistir.

A pesar de que la incidencia de endocarditis es predominante en la población adulta, el caso reportado por Nguyen et al..$^{12}$ evidencia la posibilidad de su ocurrencia en neonatos y lactantes. Por ello, al paciente descrito en este reporte se le realizó un ecocardiograma que descartó alteraciones cardiacas.

La identificación de este microorganismo se realiza por medio de su aislamiento en LCR, sangre, fluidos u otros tejidos. El análisis del hemograma y la celularidad del LCR son inespecíficos y no resultan de utilidad para identificar el agente. Un estudio francés sobre datos de pacientes con infecciones por S. gallolyticus sugirió que los valores de proteína $C$ reactiva eran más elevados que los detectados con otros patógenos; sin embargo, la evidencia de esto aún no es clara ${ }^{5,9}$. En el presente caso, la celularidad del LCR fue inespecífica debido a un cuadro de meningitis bacteriana. El crecimiento del patógeno en el LCR se obtuvo utilizando métodos automatizados, aunque los hemocultivos resultaron negativos para S. gallolyticus, por lo que se llegó al diagnóstico final de meningitis por S. gallolyticus subsp. pasteurianus.

El medicamento de elección para las infecciones sistémicas por S. gallolyticus es la penicilina $\mathrm{G}$ y sus derivados; en caso de alergia se recomienda el uso de vancomicina. Hay otros fármacos que logran una adecuada respuesta en las infecciones por estreptococos del grupo D, como la daptomicina, el linezolid y la tigeciclina, que se reservan para uso exclusivo en caso de intolerancia a los betalactámicos y la vancomicina. Existe resistencia a las tetraciclinas y los macrólidos, y no se recomienda el uso de trimetoprima-sulfametoxazol ni de clindamicina ${ }^{5}$. Con respecto a la cobertura empírica con gentamicina y penicilina, se decidió utilizar de manera inicial la terapia dual, ya que no se contaba en ese momento con los reactivos necesarios para la sensibilidad antibiótica correspondiente en el equipo utilizado.

Por lo general, el grupo de S. gallolyticus presenta un buen pronóstico en cuanto a su resolución y baja tasa de mortalidad ${ }^{3}$. La infección por este patógeno puede provocar complicaciones como neumonitis, ventriculitis, hemorragia intraventricular, derrame subdural o secuelas neurológicas tardías, como movimientos tónico-clónicos y fiebre ${ }^{1,3,5,12,14}$. En este caso, el paciente mostró una óptima respuesta al tratamiento proporcionado y, consecuentemente, no se documentaron secuelas.

La meningitis bacteriana es una causa importante de morbimortalidad infantil. Los agentes del grupo $D$ de los estreptococos causan alrededor del $5 \%$ de estos casos, y S. gallolyticus subsp. pasteurianus suele ser el más frecuentemente asociado con meningitis y sepsis neonatal ${ }^{5}$. Esta infección puede semejar cuadros de sepsis y meningitis por $S$. agalactiae, que es sumamente frecuente en la patología infecciosa neonatal, por lo que una correcta identificación del agente permite tratar con prontitud la infección con la terapia adecuada y evitar complicaciones o secuelas a largo plazo.

Existen pocos casos documentados sobre infecciones por S. gallolyticus en la población pediátrica. Debido a que la presentación clínica es muy similar a 
la de las infecciones por estreptococos del grupo B, suele subdiagnosticarse. El cultivo del LCR permite la interpretación exacta del agente; sin embargo, una limitante para identificar este microorganismo puede ser la falta del equipo y de los reactivos adecuados.

A pesar de ser una infección que puede tratarse con el debido régimen antibiótico, la escasez de información en la literatura dificulta la existencia de un protocolo específico a seguir al día de hoy.

El reporte de casos como el que se presenta contribuye a dilucidar la importancia de considerar a $S$. gallolyticus como causa de meningitis bacteriana, en especial en neonatos y lactantes pequeños, a fundar las bases para una mejor comprensión de la fisiopatología que causa y poder estandarizar los adecuados manejo y tratamiento de las infecciones producidas por esta bacteria.

\section{Responsabilidades éticas}

Protección de personas y animales. Los autores declaran que para esta investigación no se han realizado experimentos en seres humanos ni en animales.

Confidencialidad de los datos. Los autores declaran que han seguido los protocolos de su centro de trabajo sobre la publicación de datos de pacientes.

Derecho a la privacidad y consentimiento informado. Los autores han obtenido el consentimiento informado de los pacientes o sujetos referidos en el artículo. Este documento obra en poder del autor de correspondencia.

\section{Conflicto de intereses}

Los autores declaran no tener ningún conflicto de intereses.

\section{Financiamiento}

Ninguno.

\section{Bibliografía}

1. Hoen B. Infections due to group D streptococci (Streptococcus bovis/ Streptococcus equinus complex). UpToDate. Waltham, MA; 2019. (Consultado el 30 de mayo de 2019.) Disponible en: https://www.uptodate. com/contents/infections-due-to-group-d-streptococci-streptococcus-bovis-streptococcus-equinus-complex

2. Dekker JP, Lau AF. An update on the Streptococcus bovis group: classification, identification, and disease associations. J Clin Microbiol. 2016;54:1694-9

3. Park J, Eun S, Kim E, Seong M, Kim Y. Neonatal invasive Streptococcus gallolyticus subsp. pasteurianus infection with delayed central nervous system complications. Korean J Pediatr. 2015;58:33-6.

4. Beneteau A, Levy C, Foucaud P, Béchet S, Cohen R, Raymond J, et al. Childhood meningitis caused by Streptococcus bovis group: clinical and biologic data during a 12-year period in France. Pediatr Infect Dis J. 2015;34:136-9.

5. Hede S. Diagnosis and treatment of childhood meningitis caused by Streptococcus bovis group. Curr Infect Dis Rep. 2016;18:1419-22.

6. Clarridge JE, Attorri SM, Zhang Q, Bartell J. 16 S ribosomal DNA sequence analysis distinguishes biotypes of Streptococcus bovis: Streptococcus bovis biotype II/2 is a separate genospecies and the predominant clinical isolate in adult males. J Med Microbiol. 2001;39:1549-52.

7. Gavin P, Thomson R, Horng SJ, Yogev R. Neonatal sepsis caused by Streptococcus bovis variant (biotype II/2): report of a case and review. J Med Microbiol. 2003;41:3433-5.

8. Onoyama S, Ogata R, Wada A, Saito M, Okada K, Harada T. Neonatal bacterial meningitis caused by Streptococcus gallolyticus subsp. pasteurianus. J Med Microbiol. 2009;58:1252-4.

9. Floret N, Bailly P, Thouverez M, Blanchot C, Alez-Martin D, Menget A, et al. A cluster of bloodstream infections caused by Streptococcus gaIlolyticus subspecies pasteurianus that involved 5 preterm neonates in a university hospital during a 2-month period. Infect Control Hosp Epidemiol. 2010;31:194-6.

10. Klatte JM, Clarridge JE, Bratcher D, Selvarangan R. A longitudinal case series description of meningitis due to Streptococcus gallolyticus subsp. pasteurianus in infants. J Clin Microbiol. 2012;50:57-60.

11. Vélez-Balestro L, Baroni M, Ochoteco M, Zurbriggen M, Virgolini S. Streptococcus gallolyticus subsp. pasteurianus en líquido cefalorraquídeo de un paciente pediátrico. Rev Argen Microbiol. 2013;45:254-6.

12. Nguyen MT, Idriss S, Guzman E, De Oliveira ER. Neonatal meningitis, endocarditis, and pneumonitis due to Streptococcus gallolyticus subsp. pasteurianus: a case report. BMC Pediatrics. 2019:19:1-5.

13. Doran KS, Benoit VM, Gertz RE, Beall B, Nizet V. Late-onset group B streptococcal infection in twins: insight to disease pathogenesis. J Perinatol. 2002;22:326-30.

14. Yakamura $Y$, Mihara $Y$, Nakatani $K$, Nishiguchi T, Ikebe T. Unexpected ventriculitis complication of neonatal meningitis caused by Streptococcus gallolyticus subsp. pasteurianus: a case report. Jpn J Infect Dis. 2018;71:68-71. 